\title{
Laser graphitization for polarization of diamond sensors
}

\section{G. Parrini 1}

Università di Firenze e INFN Firenze

Via G. Sansone 1, 50029 Sesto Fiorentino (FI), Italy

E-mail: parriniefi.infn.it

\section{F. Fabbrizzi}

Università di Firenze e INFN Firenze

Via G. Sansone 1, 50029 Sesto Fiorentino (FI), Italy

E-mail: ilfarbi@gmail.com

\section{S. Lagomarsino}

Università di Firenze e INFN Firenze

Via G. Sansone 1, 50029 Sesto Fiorentino (FI), Italy

E-mail: lagomarsino@fi.infn.it

\section{Nunziati}

Università di Firenze e INFN Firenze

Via G. Sansone 1, 50029 Sesto Fiorentino (FI), Italy

E-mail: Iorenzonunziati@gmail.com

\section{S.Sciortino}

Università di Firenze e INFN Firenze

Via G. Sansone 1, 50029 Sesto Fiorentino (FI), Italy

E-mail: sciortinoefi.infn.it

\section{A. Scorzoni}

Università degli Studi di Perugia and INFN Perugia

Via G.Duranti 93, 06125 Perugia, Italy

E-mail:andrea. scorzoni@diei.unipg. it

We present the performances of a polycrystalline diamond detector with surface graphite electrodes when irradiated by a beta source. The results are compared with those obtained with a similar diamond detector equipped with standard Ti-Au electrodes. Both the test apparatus for signal acquisition and the laser system for the graphite electrodes are described. The quite good results open the way to a smart technique for diamond electrodes which can fit several issues as those of the CHIPSODIA R\&D program here shortly outlined.

10th International Conference on Large Scale Applications and Radiation Hardness of Semiconductor Detectors Firenze, Italy

July 6-8, 201

\footnotetext{
1 Speaker
} 


\section{Introduction}

This work is part of the program which has been recently started by the INFN (Firenze, Perugia, Bari) and IIT (Genova) researchers in the frame of the CHIPSODIA collaboration [1]. Its aims stem from the availability of the laser technique [2], [3] according to which it is possible to solder Silicon and Diamond (Dia) dies whatever their material quality is.

The main goal is the integration of electronics chips (Silicon) with Diamond dies to build monolithic rugged Silicon-on-Diamond (SoD) devices for various fields of applications. Diamond can be either the sensor of a radiation detector or the support of a high density multi electrode array (MEA) for neuronal tissue. In both cases (Figure 1) the electronics detects signals from Dia side by means of conductive Through Silicon Vias (TSV). In both cases post bonding processes are required to electrically contact TSVs at the Si-Dia interface. In the detector case the buried contacts are the sensor electrodes while in the other case they are the long probes for the neural tissue. Also in the detector case contacts can assume the shape of long probes, this is the case of a $3 \mathrm{D}$ architecture.

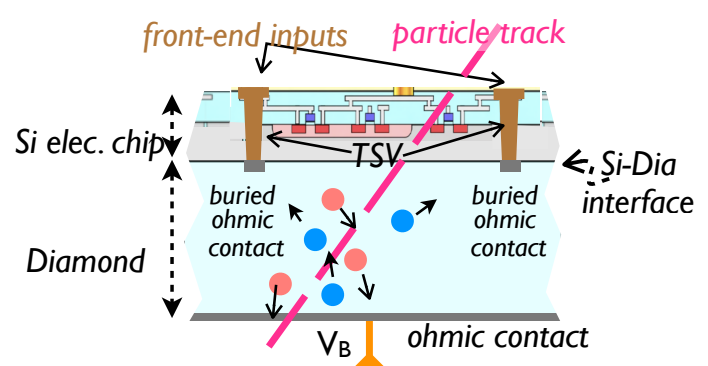

a) Planar pixel detector

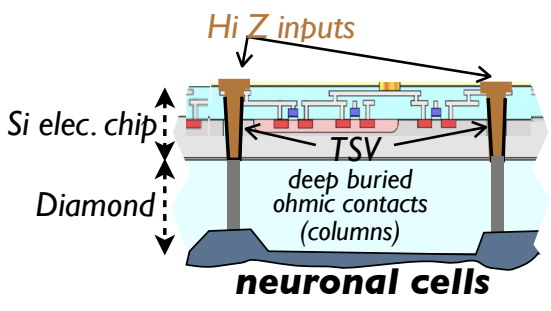

b) MEA

Figure 1 - a) The TSVs connect the electronics inputs with the buried contacts at the Si-Dia interface. b) The TSVs are extended toward the biological tissue on the free Diamond surface by means of conductive columns. In both SoD devices input pitches and thicknesses are not to scale. Expected values are: $\approx 50 \mu \mathrm{m}$ (pitch), $\approx 50 \div 500 \mu \mathrm{m}$ (Diamond thickness) and $\leq 50 \mu \mathrm{m}$ (Si thickness).

In the present work we address the issue of the laser graphitization of Diamond. Graphite is a conductor $(\approx 1 \div 500 \mathrm{~m} \Omega$.cm) and the laser process can be the elegant and simple solution for the buried contacts fabrication if using wavelengths above the Diamond absorption threshold $(\approx$ $225 \mathrm{~nm}$ ) and focusing the beam inside Diamond in the due place. This technique can be used in both types of SoD devices. Graphitization is produced in the beam focus region. Several parameters of the laser system contribute to the process and they all must be accurately investigated to achieve a reliable buried contact technique. Concerns are about the integrity of diamond because of the difference of the specific volumes. Concerns are also on the quality and on the shapes of the graphite artifacts. But one issue is mandatory for detector devices: the electric quality of the Graphite-Diamond contact. Its reputation in literature is a bit controversial [4], [5] and for that we have investigated the voltage biasing of a poly CVD detector equipped with surface graphite electrodes.

The characteristics of the laser system, of the graphitization process and of the graphite biasing electrodes are described in the following sections. The behavior of the graphitized detector with respect to a reference detector with standard surface electrodes is presented and 
discussed as well.

The detectors were implemented on electronic grade poly CVD samples from Diamond Detector Ltd. The two samples have volume of $\approx 5 \times 5 \times 0.5 \mathrm{~mm}^{3}$, surface roughness about $10 \mathrm{~nm}$ or less and grain size of $50 \div 100 \mu \mathrm{m}$ on both sides. Since the surfaces is highly polished and the roughness well below the wavelength of the laser beam we believe that the surface radiation scattering was negligible in the graphitizing process. We also believe that the influence of the grain boundaries, even if not known, is not critical for the graphitization process. Few graphitization tests performed on a single crystal CVD sample showed only energy threshold effects.

One of the two diamond samples was equipped with standard Ti-Au surface electrodes by the CNR-IMM facility and the other with graphite surface patterns as it will be explained in the next section. The two detectors were exposed to a beta source with the same set-up for a comparison. The obtained results show the substantial equivalence of the graphite electrodes to the Ti-Au ones at least on the short term scale (few months).

\section{Laser graphitization of Diamond}

With the laser wavelength of Figure 2b) the graphitization process occurs inside the focus spot if the pulse energy exceeds a minimum value. This value depends on the focus position inside the poly diamond sample. With our laser at $10 \mathrm{kHz}$, if we focus on a surface in contact

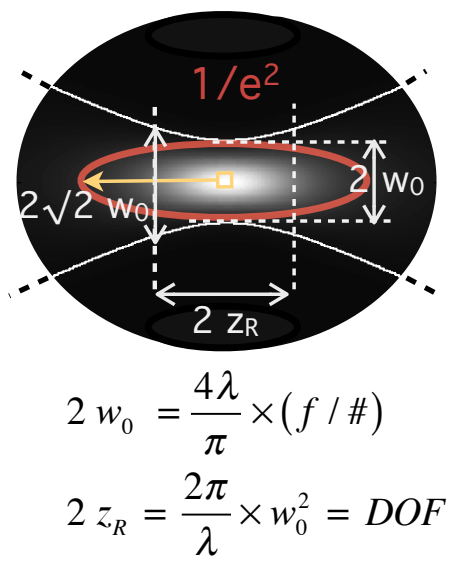

a)

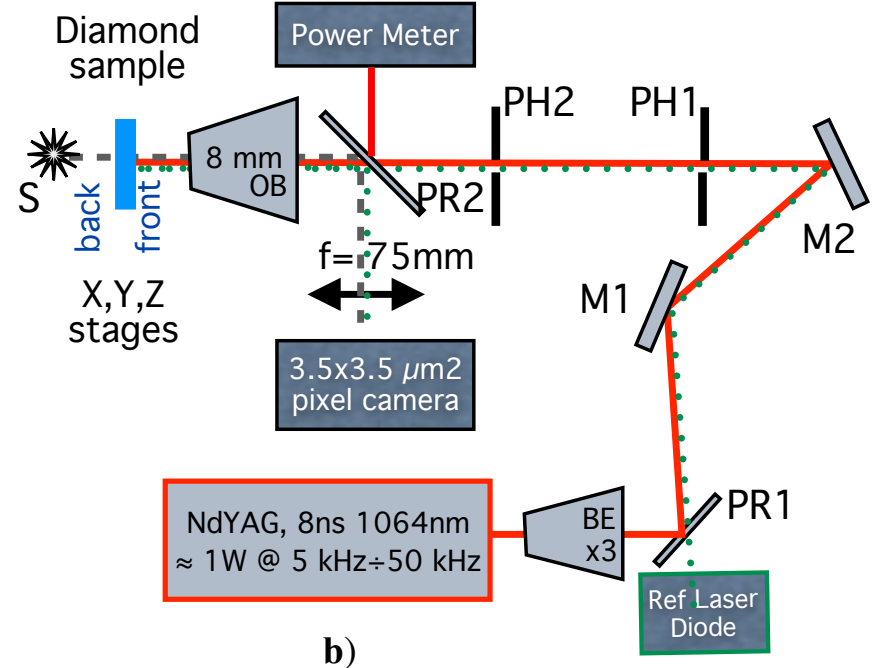

b)

Figure 2 - a) The beam focus region is enclosed by the $1 / \mathrm{e}^{2}$ maximum irradiance red surface. Its sizes are given by the "waist" $\mathrm{w}_{0}$ and by the Depth Of Focus (yellow arrow). $\mathrm{f} /$ /\# is the stop number of the optics. b) The NdYAG beam (red line) is expanded at $\varnothing \approx 1.5 \mathrm{~mm}$ and then it is focused by the objective (OB) on the Dia sample. The beam crosses the sample from the "front" to the "back" side. The X, Y, Z automatic stages hold the sample. PR are beam splitters used also to overlap the source S and Ref. Laser Diode auxiliary paths to that of the laser. M, PH are mirrors and pin holes respectively. The $75 \mathrm{~mm}$ lens is the pixel camera objective. 
with an absorbing material we find the minimum of $\approx 7 \mathrm{~J} / \mathrm{cm}^{2}$.pulse, otherwise we find a threshold at least a factor two higher. The former case comprehends SoD interfaces, the latter the graphitization on free surfaces as we will describe in the following.

The quality of the graphitic artifacts depends on several parameters of the process but of basic importance is the geometry of the focus spot and the energy distribution inside it. The geometry can be described by the waist ( $\left.\mathrm{w}_{0}\right)$ and DOF parameters as it is shown in Figure 2 a) for a gaussian beam [6]. Anyway to take into account the graphitization threshold effects the Rayleigh parameter $\mathrm{z}_{\mathrm{R}}$ should be more appropriate to indicate the effective depth of the focus. In our system the expected focus dimension are $2 \mathrm{w}_{0} \approx 7 \mu \mathrm{m}, 2 \mathrm{zR}_{\mathrm{R}} \approx 76 \mu \mathrm{m}$ (DOF) in air, while inside diamond only the longitudinal dimension increases by the index of refraction $(\approx 2.4)$ giving DOF $\approx 182 \mu \mathrm{m}\left(z_{R} \approx 91 \mu \mathrm{m}\right)$. Smaller sizes can be obtained reducing wavelength and stop number. In our case the latter could be modified by simply changing the Beam Expander.

Experimentation on column manufacturing is now in progress. Either in the case of SoD or in that of a Dia sample the graphitization process is performed by starting from the "back" side of diamond and going towards the "front" one. Figure 3 shows three columns crossing a $500 \mu \mathrm{m}$ thick sample. Mean diameter (Ø), section (S), resistance (R) and resistivity ( $\rho$ ) of each column are reported in the table together with the used energy (E) per pulse [7]. The variation of the resistivity is due to the poor regularity of the cross sections which is to improve by a better control of the graphitization process.

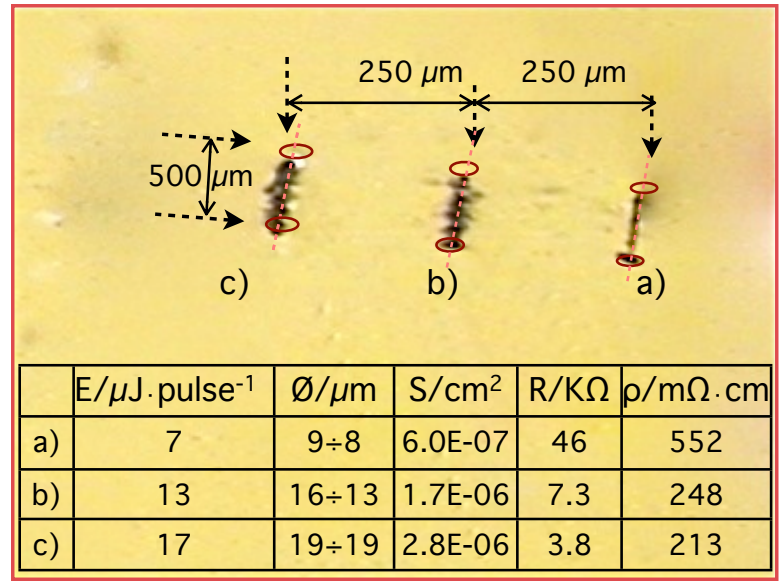

Figure 3 - Three graphite columns cross the poly diamond sample. The microscope picture has been taken with the sample tilted of $\approx 60^{\circ}$. Red circles and dotted lines are for guiding the eye. The misleading apparent longitudinal dimension is due also to the high refraction index of diamond. For the table see text.

\subsection{Surface laser graphitization of Diamond}

With the laser system of Figure $2 \mathrm{~b}$ ) we carved each surface of one of the poly diamond samples with a long zigzag (Greek Fret like) graphite track which spans a large part of the sample area (Figure 4). The GF patterns on the two sides of the sample are similar and orthogonal to each other in order to maximize their coupling.

The graphite track was obtained focusing the beam $(10 \mathrm{kHz}, 20 \mu \mathrm{J} / \mathrm{pulse})$ on the front side of the sample and moving it $(250 \mu \mathrm{m} / \mathrm{s})$ according to the desired GF pattern. Both sides of the 
sample were processed independently with the same method. The result was $\approx 9 \mu \mathrm{m}$ wide and $\approx$ $55 \mu \mathrm{m}$ deep tracks which recall the size of the laser focus in Diamond and the discussion on its effective depth. As it is shown in Figure 4, the track geometry is very well defined.

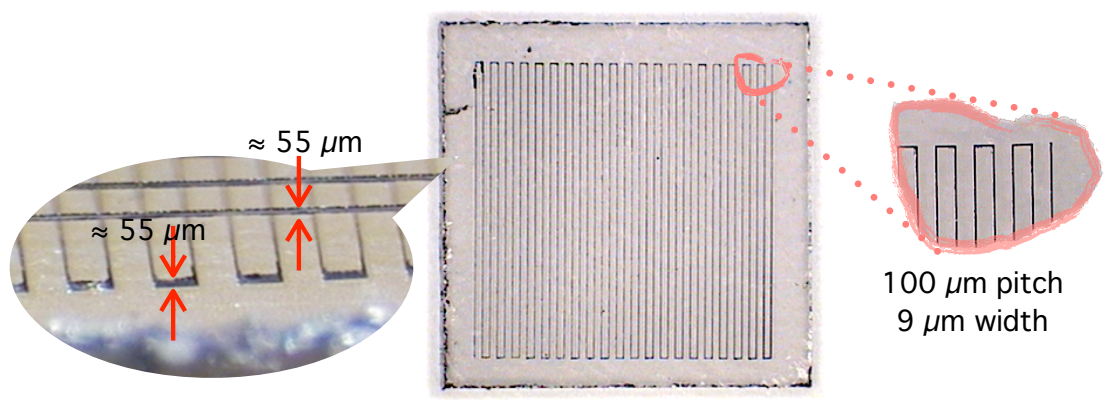

Figure 4 - The $100 \mu \mathrm{m}$ pitch and $9 \mu \mathrm{m}$ wide Greek Fret track is carved on the $4 \times 4 \mathrm{~mm}^{2}$ central area of a poly diamond die of $5 \times 5 \times 0.5 \mathrm{~mm}^{3}$ volume. The regularity of the track is visible in the two enlarged portions. On the left one the partial vision of the orthogonal tracks of the opposite surfaces shows their thickness $(\approx 55 \mu \mathrm{m})$. The picture was taken with the die tilted at $\approx 45^{\circ}$.

The graphite electrical behavior is shown by the measurements of Figure 5 a). The fairly good linearity of the resistance as a function of the track length as well as the good resistivity value obtained agree with the good quality of the track as is highlighted by optical inspection. The resistivity was calculated from the measured track depth and width and assuming an elliptical cross section. The obtained result is $\rho=4.4 \mathrm{~m} \Omega \cdot \mathrm{cm} \pm 20 \%$. The track depth was estimated by measuring the apparent track thickness while varying the sample inclination with respect to the microscope axis.

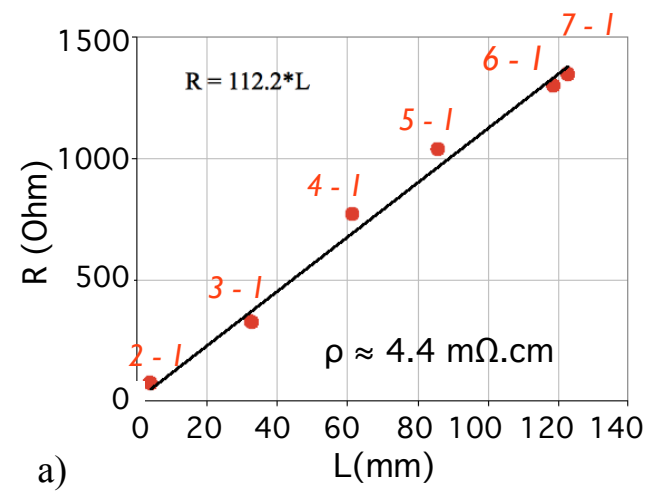

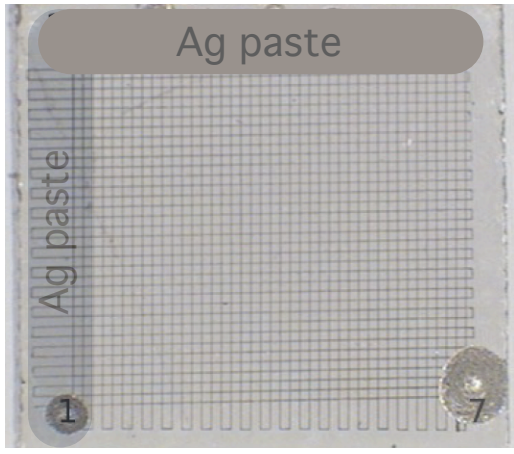

b)

Figure 5 - a) Electric resistance of a track versus its length. 1,7 are the two extremities of the GF track. The other numbers refer to intermediate points of the track. b) The GF detector with the "Ag paste" strips glued on the two sides. The overlap between the two electrodes (Ag paste strips included) is $\approx 59 \%$ of the sample surface.

The GF conductive patterns can be used to polarize the diamond sample in order to study the charge collection efficiency. However their depth reduces the effective thickness of the sensor by $\approx 110 \mu \mathrm{m}$ while their overlap assures a fairly uniform electric field in the bulk.

These limitations are not important at this stage of our experimentation, however they can be limited by reducing the focus size and by choosing a suitable geometry of the pattern to maintain a low total resistance. In order to bias the structure, a wide silver paste strip is glued on 
one side of each face of the diamond (Figure $5 \mathrm{~b}$ )). This assures a good potential uniformity of the GF track together with a fairly low resistance of the electrodes $(<30 \Omega)$.

\section{Comparison between poly diamond detectors}

The GF detector was tested together with the detector which was built on the second poly CVD sample by covering the $\approx 85 \%$ of its faces with very thin Ti-Au electrodes. The Ti-Au detector has an active thickness of $500 \mu \mathrm{m}$ and its measured capacitance is $(2.40 \pm 0.05) \mathrm{pF}$. The GF detector active area is $\approx 59 \%$. of the die surface and its capacitance is $(2.07 \pm 0.05) \mathrm{pF}$ which gives $\approx 400 \mu \mathrm{m}$ for the active thickness, in well enough agreement with the $\approx 390 \mu \mathrm{m}$ we estimated from the depth of the graphite tracks.

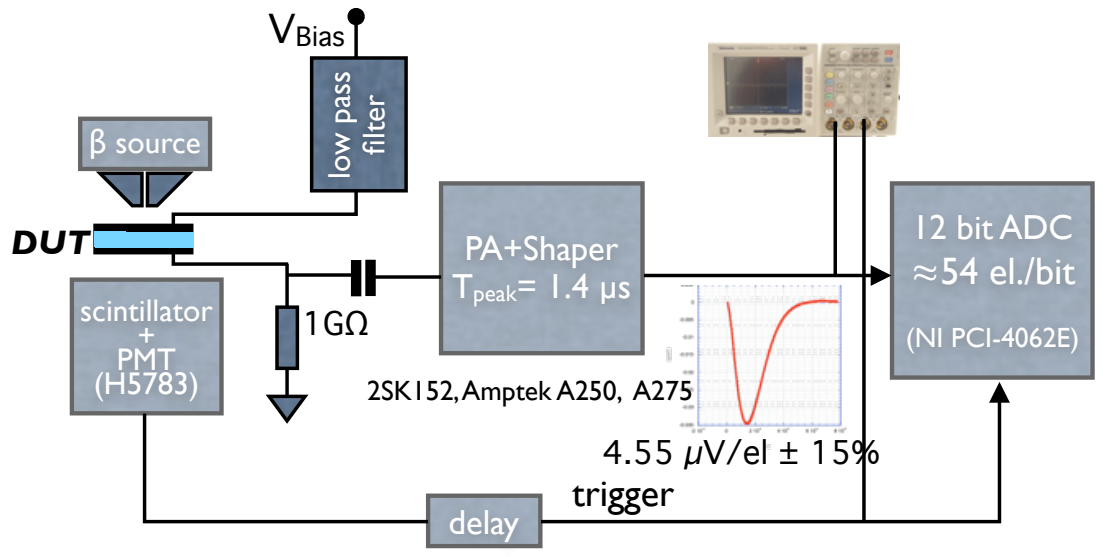

Figure 6 - The set-up used to test diamond detectors. The red curve is the pulse shape peaked at $1.4 \mu$ s. Its amplitude is negative when $\mathrm{V}_{\text {Bias }}$ is positive and vice versa. The ADC calibration is used for CCE calculation (see text). Not shown the pulse generator used for random triggers.

The detectors were tested with the set-up of Figure 6 where a small ${ }^{90} \mathrm{Sr}$ beta source irradiates the detector under test through a collimator with $\mathrm{a} \approx 0.6 \mathrm{~mm}$ aperture [8]. Beta particles which reach the scintillator behind the detector generate the acquisition trigger.
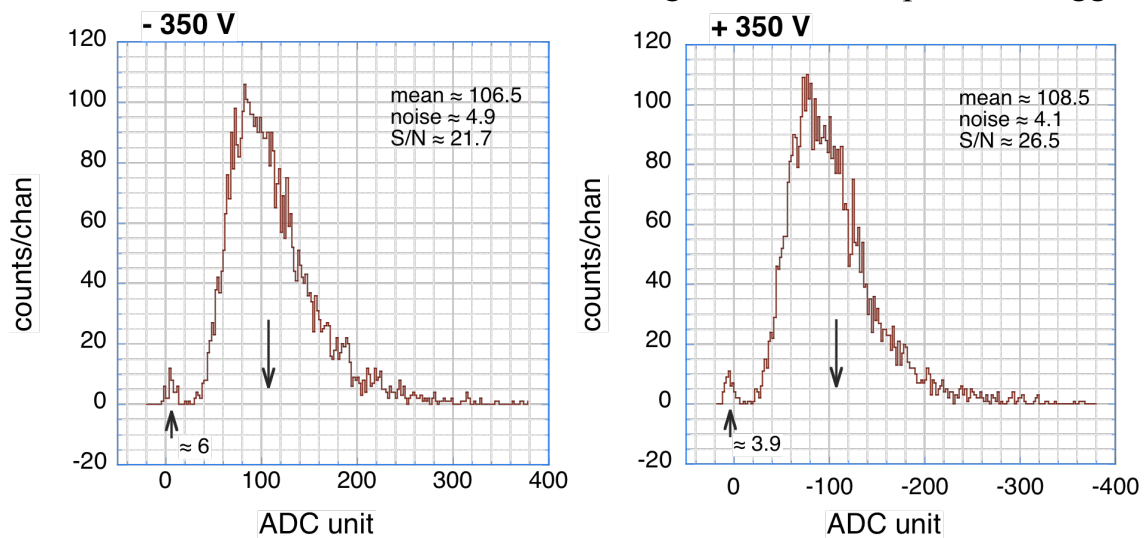

Figure 7 - The straggling of the beta signals in the GF detector at the two polarizations of \pm 350 V. The two histograms have about 4500 acquisitions each. The small arrows indicate the base line of the signals, the large arrows the means. 
The experimented trigger rate was $\approx 10 \mathrm{~Hz}$. The acquisition is performed by a 12 bit ADC housed in a PC slot. The main parameters of the front-end electronics are reported in Figure 6. This is based on hybrid commercial circuitry buffered by an input FET with a noise resistance of about $150 \Omega$. This value is sufficiently larger than that estimated for the GF detector contacts. Figure 7 shows the amplitudes of beta signals generated by the GF detector in two different runs performed with symmetric bias voltages chosen at the beginning of the asymptotic region of the detector. The two runs accumulated about the same statistics. The small peaks near the zero of the scale are due to false triggers and they give an indication of the position and of the width (noise) of the base line of the signals. The two amplitude distributions are quite similar and have their signal to noise ratios well above 20. The dependence of the amplitude of signals as a function of the bias voltage was studied for the two detectors and the main results are synthesized in Figure 8.
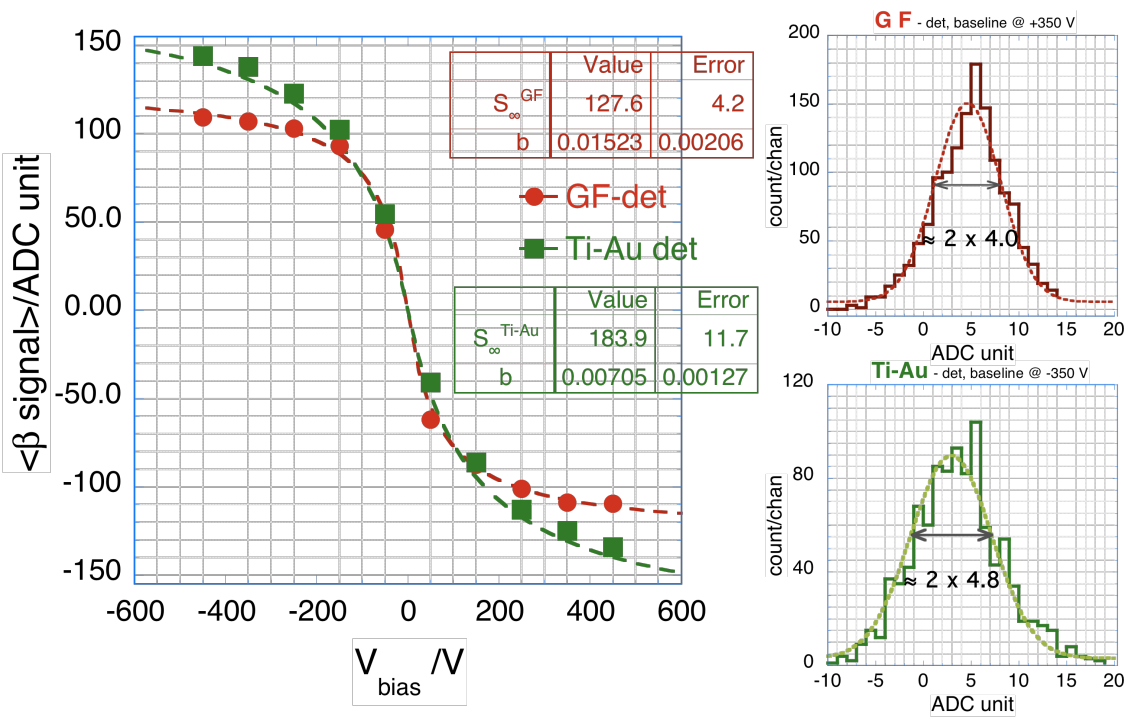

Figure 8 - The large plot shows the means of the beta signals and their fitted parameters (see text). The statistical uncertainties of the data are within the symbol dimensions. The two histograms on the right show the distribution of random output samples and their fit with a gaussian function for the two detectors. Their mean values define the pedestal of the electronics, their width its noise. The quoted values are the standard deviations of the fits.

The mean beta signals of the larger plot are the averages of amplitudes acquired in runs of about 2000 events each. The two behaviors are quite symmetric with respect to the bias voltage sign, they rise sharply until few hundred volts and then flatten heading asymptotic values. This effect is mostly due to the mean free path of the diamond charge carriers and to their mobility which, at high electric strength values, decreases as the electric field increases. Experimental data have been fitted with the drift velocity like function [9] $S=S_{\infty} \cdot\left(b \cdot V_{\text {bias }}\right) /\left(1+\left|b \cdot V_{\text {bias }}\right|\right)$, where $S_{\infty}$ is the asymptotic value and $b$ is a scale factor. The fits show that the GF detector is at $\approx 80 \%$ of its asymptotic value ( $S_{\infty}{ }^{G F} \approx \pm 128 \mathrm{ADC}$ unit) at $\approx \pm 260 \mathrm{~V}$ while the Ti-Au one is at the same fraction of its asymptotic value $\left(S_{\infty}^{T i-A u} \approx \pm 184\right.$ ADC unit) at $\approx \pm 567 \mathrm{~V}$. The two bias voltages correspond to the same $b \cdot V_{\text {bias }}$ value. It is interesting to note that $S_{\infty}{ }^{T i-A u} / S_{\infty}{ }^{G F} \approx 1.44$ which is very close to $t^{T i-A u} / t^{G F} \approx 1.28$, the ratio of the sensor volume active thicknesses. The estimated 
uncertainties of the two ratios are respectively $\approx 7 \%$ and $\approx 1.5 \%$ which show the importance of increasing the experimental points for a better resolution. Also with not asymptotic signals taken at corresponding voltages (same $b \cdot V_{\text {bias }}$ ) their ratio is about the ratio of the active thicknesses of the detectors. Recalling the Charge Collection Efficiency (CCE) quantity [10] which is given by the ratio of the collected charges (see ADC calibration of Figure 7) with those delivered by betas passing through the sensor (36 electrons per micron of active thickness), our experimental data indicate that the CCEs of the detectors are very close and that the CCE of the GF detector is $\approx$ $12 \%$ lower the that of Ti-Au device at corresponding bias voltages. The difference is less relevant if we bias the detectors according to the commonly used criteria of $\approx 1 \mathrm{~V}$ per micron of thickness [11]. In such conditions the CCE of GF detector becomes $\approx 0.42$ while that of the Ti$\mathrm{Au}$ one $\approx 0.43$. In conclusion we have found that the large part of the collected charges is proportional to the active volume thickness while only a small part may depend on either the graphite-diamond interface physics or the diamond sample quality or both. The comparison of the pedestal runs of Figure 8 and their similar shapes suggests that the graphite-diamond interface is not involved, but this must be better investigated.

\section{Conclusions}

We have compared the characteristics of two poly CVD detectors based on samples of the same geometry and of the same quality as it is specified by the Diamond Detector Ltd. The two samples have been equipped with bias surface electrodes of different geometry and different techniques, the standard Ti-Au contact process and the laser surface graphitization without metal covering. The graphitization has been done by using a nanosecond laser at $1064 \mathrm{~nm}$ with the drawback of reducing the total thickness of the sensor volume. However this limitation is not intrinsic to the laser technique, it is only due to the experimental set-up used in this work.

When exposed to a ${ }^{90} \mathrm{Sr}$ beta source the two detectors show a small difference $(\approx 12 \%)$ in their CCEs which should be confirmed by more extended measurements. The difference can be due to either marginal quality variations or the graphite-diamond interface or both even if the substantial invariance of the electronic noise in the two cases suggests the first of the three hypothesis.

The results obtained require to go ahead in the experimentation but the conclusion is the feasibility of the laser graphite contacts on diamond to collect particle signals and its flexibility not only for the buried SoD contacts of CHIPSODIA devices but also for wider applications.

\section{Acknowledgments}

The authors thank the technological department of CNR-IMM Bologna, Italy, and in particular Dr. P.Maccagnani and Dr. I.Elmi for the preparation of the Ti-Au samples

\section{References}

[1] S. Sciortino et al., New perspectives for the Silicon-On-Diamond material, in proceedings of 9th Int. Conf. on Large Scale Appl. and Rad. Hard. of Semi. Det. 30 September - 2 October 2009, Florence, Italy, PoS (RD09) 029 
[2] S. Lagomarsino et al., Silicon-on-diamond material by pulsed laser technique,Appl. Phys. Lett. 96,(2010) 031901

[3] S. Lagomarsino, Phenomenological model of Silicon-On-Diamond laser bonding, Diam. Relat. Matem. 20 (2011) 1010-1015.

[4] T. Tachibana, J. T. Glass, Electric contacts to diamond,"Diamond: Electronic Properties and Applications (Electronic Materials: Science \& Technology)", L. S. Pan, D. R. Kania, Editors, Springer; 1 edition (Dec. 31, 1994)

[5] M.W.Geis,et al., Electrical, crystallographic, and optical properties of ArF laser modified diamond surfaces, Appl. Phys. Lett. 55,(1989) 2295 - 2297

[6] .A.Yariv , P. Yeh, Photonics: Optical Electronics in Modern Communications (The Oxford Series in Electrical and Computer Engineering), January 26, 2006, ${ }^{\text {th }}$ edition, ISBN-10: 0195179463.

[7] Writing unit symbols and names http://www.bipm.org/utils/common/pdf/si brochure 8 en.pdf

[8] S. Sciortino, et al., Silicon Carbide for High Signal to Noise Ratio MIPs Detection From Room Temperature to $80^{\circ}$, IEEE Trans. Nucl. Sci. 56, no. 4, suppl. 3 (2009) $2538-2542$.

[9] M.Gabrysch, S.Majdi, D.J Twitchen, J.Isberg, Electron and hole drift velocity in chemical vapor deposition diamond, J. Appl. Phys. 109, 063719 (2011

[10] A. Lohstroh et al., The effect of fast neutron irradiation on the performance of synthetic single crystal diamond particle detectors, Diamond \& Related Materials 19 (2010) 841-845

[11] The RD42 Collaboration Development of Diamond Tracking Detectors for High Luminosity Experiments at the LHC CERN/LHCC 2008-005 LHCC-RD-016 Status Report/RD42 April 4, 2008 